\title{
Real-time intelligent image processing for security applications
}

\author{
Akansha Singh ${ }^{1} \cdot$ Ping $^{L^{2}} \cdot$ Krishna Kant Singh ${ }^{3} \cdot$ Vijayalakshmi Saravana ${ }^{4}$ \\ Published online: 5 September 2021 \\ (c) The Author(s), under exclusive licence to Springer-Verlag GmbH Germany, part of Springer Nature 2021
}

\begin{abstract}
The advent of machine learning techniques and image processing techniques has led to new research opportunities in this area. Machine learning has enabled automatic extraction and analysis of information from images. The convergence of machine learning with image processing is useful in a variety of security applications. Image processing plays a significant role in physical as well as digital security. Physical security applications include homeland security, surveillance applications, identity authentication, and so on. Digital security implies protecting digital data. Techniques like digital watermarking, network security, and steganography enable digital security.
\end{abstract}

\section{Accepted papers}

The rapidly increasing capabilities of imaging systems and techniques have opened new research areas in the security domain. The increase of cyber and physical crimes requires novel techniques to control them. In the case of both physical and digital security, real-time performance is crucial. The availability of the right image information at the right time will enable situational awareness. The real-time image processing techniques can perform the required operation by a latency being within the required time frame. Physical security applications like surveillance and object tracking will be practical only if provided in real time. Similarly, biometric authentication, watermarking or network security is also time restricted applications and requires realtime image processing. This special issue aims to bring together researchers to present novel tools and techniques

Akansha Singh

akanshasing@gmail.com

Krishna Kant Singh

krishnaiitr2011@gmail.com

1 Computer Science Engineering Department, Bennett University, Greater Noida, India

2 Department of Computing, The Hong Kong Polytechnic University, Kowloon, Hong Kong

3 Faculty of Engineering and Technology, Jain (Deemed-To-Be University), Bengaluru, India

4 Department of Computer Science, University of South Dakota, Vermillion, USA for real-time image processing for security applications augmented by machine learning techniques.

This special issue on Real-Time Intelligent Image Processing for Security Applications comprises contributions on the topics in theory and applications related to the latest developments in security applications using image processing. Real-time imaging and video processing can be used for finding solutions to a variety of security problems. The special issue consists of the articles that address such security problems.

The paper entitled "RGB $+\mathrm{D}$ and deep learning-based real-time detection of suspicious event in Bank ATMs" presents a real-time detection method for human activities. The method is applied to enhance the surveillance and security of Bank Automated Teller Machine (ATM) [1]. The increasing number of illicit activities at ATMs has become a security concern.

The existing methods for surveillance involving human interaction are not very efficient. The human surveillance methods are highly dependent on the security personnel's behavior. The real-time surveillance of these machines can be achieved by the proposed solution. The authors have presented a deep learning-based method for detecting the different kinds of motion from the video stream. The motions are classified as abnormal in case of any suspicious activity.

The paper entitled "A real-time person tracking system based on SiamMask network for intelligent video surveillance" presents a real-time surveillance system by tracking persons. The proposed solution can be applied to various public places, offices, buildings, etc., for tracking persons 
[2]. The authors have presented a person tracking and segmentation system using an overhead camera perspective.

The paper entitled "Adaptive and stabilized real-time super-resolution control for UAV-assisted smart harbor surveillance platforms" presents a method for smart harbor surveillance platforms [3]. The method utilizes drones for flexible localization of nodes. An algorithm for scheduling among the data transmitted by different drones and multi-access edge computing systems is proposed. In the second stage of the algorithm, all drones transmit their own data, and these data are utilized for surveillance. Further, the authors have used the concept of super resolution for improving the quality of data and surveillance. Lyapunov optimization-based method is used for maximizing the timeaverage performance of the system subject to stability of the self-adaptive super resolution control.

The paper entitled "Real-Time Video Summarizing using Image Semantic Segmentation for CBVR" presents a realtime video summarizing method using image semantic segmentation for CBVR [4]. The paper presents a method for summarizing the videos frame-wise using stacked generalization by an ensemble of different machine learning algorithms. Also, the ranks are given to videos on the basis of the time a particular building or monument appears in the video. The videos are retrieved using KD Tree. The method can be applied to different applications for security surveillance. The authors use video summarization using prominent objects in the video scene. The summary is used to query the video for extracting the required frames. The labeling is done using machine learning and image matching algorithms.

The paper entitled "A real-time classification model based on joint sparse-collaborative representation" presents a classification model based on joint sparse-collaborative representation [5]. The paper proposes the two-phase test sample representation method. The authors have made improvements in the first phase of the traditional two set method. The second phase has an imbalance in the training samples. Thus, the authors have included the unselected training samples in modeling. The proposed method is applied on numerous face databases. The method has shown good recognition accuracy.

The paper entitled "Recognizing Human Violent Action Using Drone Surveillance within Real-Time Proximity" presents a method for recognizing human violent action using drone surveillance [6]. The authors have presented a machine-driven recognition and classification of human actions from drone videos. A database is also created from an unconstrained environment using drones. Key-point extraction is performed and 2D skeletons for the persons in the frame are generated. These extracted key points are given as features in the classification module to recognize the actions. For classification, the authors have used SVM and Random Forest methods. The violent actions can be recognized using the proposed method.

\section{Conclusion}

The editors believe that the papers selected for this special issue will enhance the body of knowledge in the field of security using real-time imaging. We would like to thank the authors for contributing their works to this special issue. The editors would like to acknowledge and thank the reviewers for their insightful comments. These comments have been a guiding force in improving the quality of the papers. The editors would also like to thank the editorial staff for their support and help. We are especially thankful to the Journal of Real-Time Image Processing Chief Editors, Nasser Kehtarnavaz and Matthias F. Carlsohn, who provided us the opportunity to offer this special issue.

\section{References}

1. Khaire, P.A., Kumar, P.: RGB+ D and deep learning-based realtime detection of suspicious event in Bank-ATMs. J Real-Time Image Proc 23, 1-3 (2021)

2. Ahmed, I., Jeon, G.: A real-time person tracking system based on SiamMask network for intelligent video surveillance. J Real-Time Image Proc 28, 1-2 (2021)

3. Jung, S., Kim, J.: Adaptive and stabilized real-time super-resolution control for UAV-assisted smart harbor surveillance platforms. J Real-Time Image Proc 17, 1-1 (2021)

4. Jain R, Jain P, Kumar T, Dhiman G (2021) Real time video summarizing using image semantic segmentation for CBVR. J RealTime Image Proc.

5. Li Y, Jin J, Chen CLP (2021) A real-time classification model based on joint sparse-collaborative representation. J Real-Time Image Proc.

6. Srivastava A, Badal T, Garg A, Vidyarthi A, Singh R (2021) Recognizing human violent action using drone surveillance within real time proximity. J Real-Time Image Proc.

Publisher's Note Springer Nature remains neutral with regard to jurisdictional claims in published maps and institutional affiliations. 